\title{
Flash-Based Database Systems: Experiences from the FlashDB Project
}

\author{
Xiaofeng Meng ${ }^{1}$, Lihua Yue ${ }^{2}$, and Jianliang $\mathrm{Xu}^{3}$ \\ ${ }^{1}$ Renmin University of China, Beijing, China \\ ${ }^{2}$ University of Science and Technology of China, Hefei, China \\ ${ }^{3}$ Hong Kong Baptist University, Hong Kong, China
}

\begin{abstract}
The new characteristics of flash memory bring great challenges in optimizing database performance, by using new querying algorithms, indexes, buffer management schemes, and new transaction processing protocols. In this talk, we will first present an overview on the FlashDB project, which was launched in 2009 and supported by the National Natural Science Foundation of China (No. 60833005). The project aims at constructing the fundamental theory and design principles of flash-based database systems including a series of key problems, such as system architecture, storage management and indexing, query processing, transaction processing, buffer management, etc. In particular, we focus on establishing a basis for data management involving flash memory, developing database management systems for flash-based SSDs, and preparing a test bed for flash-based database applications. During the past two years, we have made some achievements in buffer management [1, 2], index structures [3], storage management [4], and SSD simulation platform [5]. After a brief introduction on the current research results in the project, we will discuss some experiences and lessons concluded from the study. We will emphasize several issues that may be open up exciting avenues and influence the direction of the research within the scope of flash-based database systems.
\end{abstract}

Keywords: Flash Memory, SSD, Flash-based Database.

\section{References}

1. Tang, X., Meng, X.: ACR: An Adaptive Cost-Aware Buffer Replacement Algorithm for Flash Storage Devices. In: MDM 2010, pp. 33-42 (2010)

2. Li, Z., Jin, P., Su, X., et al.: CCF-LRU: A New Buffer Replacement Algorithm for Flash Memory. IEEE Trans. on Consumer Electronics 55(3), 1351-1359 (2009)

3. Yin, S., Pucheral, P., Meng, X.: A sequential indexing scheme for flash-based embedded systems. In: EDBT 2009, pp. 588-599 (2009)

4. Zhou, D., Meng, X.: RS-Wrapper: random write optimization for solid state drive. In: CIKM 2009, pp. 1457-1460 (2009)

5. Jin, P., Su, X., et al.: A flexible simulation environment for flash-aware algorithms. In: CIKM 2009, demo (2009) 